\title{
Clomiphene Citrate is a Safe and Effective Alternative to Testosterone Replacement in Male Hypogonadism with Type 2 Diabetes Mellitus
}

\author{
SK Singh ${ }^{1 *}$, Jitendra Chouhan ${ }^{2}$ and Rujul Jain ${ }^{2}$ \\ ${ }^{1}$ Professor, Department of Endocrinology and Metabolism, Institute of Medical Sciences, Banaras Hindu University, Varanasi \\ ${ }^{2}$ Senior Resident, Department of Endocrinology and Metabolism, Institute of Medical Sciences, Banaras Hindu University, Varanasi
}

${ }^{\star}$ Corresponding author: Prof SK Singh, Department of Endocrinology and Metabolism, Institute of Medical Sciences, Banaras Hindu University, Varanasi; E-mail: sksendocrine@yahoo.com

Received: April 28, 2021; Accepted: May 03, 2021; Published: May 10, 2021

\begin{abstract}
Aims: The study was planned to evaluate the effect of Clomiphene Citrate (CC) treatment as compared to testosterone replacement for late onset hypogoandism in with type 2 Diabetes Mellitu.

Methods: The study included 72 male patients with late onset hypogonadism (assessed by ADAM questionnaire, serum total testosterone and LH) and T2DM out of 250 patients screened. The subjects with serum testosterone in the range of 200-300 ng/dl and with serum Luteinizing hormone (LH) level $\leq 9.4 \mathrm{IU} / \mathrm{ml}$ were treated with Clomiphene Citrate $25 \mathrm{mg} /$ day (Group $1 \mathrm{~N}=40$ ). Patients with serum testosterone levels less than $200 \mathrm{ng} / \mathrm{dl}$ and serum LH $<9.4 \mathrm{IU} / \mathrm{ml}$ received testosterone every month for 3 months (Group $2 \mathrm{~N}=32$ ). The post treatment hormone estimation along with ADAM questionnaire value was evaluated 3 month after commencing treatment.

Results: ADAM symptom scores were worse in group $2(\mathrm{~N}=32)$ than group $1(\mathrm{~N}=40)$. There was a comparable increase in mean testosterone levels in both groups at 3 months $(550.16 \pm 85.05$ vs $509.72 \pm 39.18 \mathrm{ng} / \mathrm{dl} ; \mathrm{p}=0.03)$. Mean ADAM scores also decreased significantly in both the groups.

Conclusion: Treatment with clomiphene citrate in male patients with T2DM and hypogonadism showed improvement in both clinical and biochemical measures. The study suggested that clomiphene citrate might be considered as a safe and effective alternative treatment strategy for late onset hypogonadism in male patients with type $2 \mathrm{DM}$.
\end{abstract}

Keywords: Hypogonadism, Clomiphene Citrate, Testosterone replacement, Type 2 diabetes mellitus

\section{Introduction}

Prevalence of type 2 diabetes mellitus (T2DM) is increasing worldwide to reach epidemic proportions ${ }^{[1]}$. Insulin resistance (IR) of variable degree is a feature of T2DM. It is recognized that low testosterone level in men is associated with reduced insulin sensitivity and T2DM. ${ }^{[2]}$. Epidemiological studies have reported that $30 \%-50 \%$ of men with T2DM have testosterone deficiency, and up to $75 \%$ of them have sexual symptoms, particularly erectile dysfunction (ED) ${ }^{[3]}$. Dhindsa et al. demonstrated that $33 \%$ of men with type 2 diabetes had significantly lower levels of testosterone ${ }^{[4]}$.

Questionnaires have been developed to assess hypogonadism. Androgen deficiency in the aging male (ADAM) questionnaire is one of them with a reasonable sensitivity $(88 \%)$ and specificity $(66 \%)$ in the presence of low testosterone levels. ${ }^{[5]}$ In EMAS ( European Male Aging Study), subjects are classified into primary hypogonadism ( $\mathrm{LH}$ $>9.4 \mathrm{u} / \mathrm{L}, \mathrm{T}<10.5 \mathrm{nmol} / \mathrm{L})$, secondary hypogonadism $(\mathrm{LH} \leq 9.4 \mathrm{u} / \mathrm{L}$, $\mathrm{T}<10.5 \mathrm{nmol} / \mathrm{L}$ ) or compensated (primary) hypogonadism $(\mathrm{LH}>9.4$ $\mathrm{u} / \mathrm{L}, \mathrm{T} \geq 10.5 \mathrm{nmol} / \mathrm{L}){ }^{[6]}$

Until very recently, treatment options mainly consisted of testosterone replacement using a variety of modalities but exogenous testosterone has unacceptable side effects. In many small studies clomiphene citrate (CC) has shown promising efficacy. As a selective estrogen receptor modulator (SERM), it acts on the Hypothalamic Pituitary - Gonadal (HPG) axis and increases gonadotrophin levels. In turn this also stimulates testosterone production and corrects androgen deficiency but its role improve hypogonadal symptoms in males with T2DM are lacking. ${ }^{[7]}$ The present study was planned to evaluate the effect of treatment with clomiphene citrate as compared to testosterone replacement for hypogonadism in patients with T2DM, based on both symptoms and biochemical measures.

\section{Subjects}

This study was conducted in the Department of Endocrinology and Metabolism, Sir Sunderlal Hospital, Institute of Medical Sciences, BHU, Varanasi. Study subjects were enrolled from April 2016 to June 2017. Subjects with T2DM with biochemical (Testosterone $<300 \mathrm{ng} / \mathrm{dl}$ with low $\mathrm{LH}<9.4 \mathrm{IU} / \mathrm{ml}$ ) and symptomatic hypogonadism, aged 40 70 years with no serious concurrent medical conditions were enrolled. Patients were included in the study after obtaining their informed consent. The exclusion criteria were subjects on drug treatment which might cause HPG axis suppression, subjects with history of tumor, exposure to radiation, history of head trauma, spinal cord injuries, 
and history of pelvic trauma or with chronic disease such as human immunodeficiency virus, end-stage renal disease, liver cirrhosis, and psychiatric disease. A detailed clinical evaluation was done and recorded.

A total of 250 patients underwent screening for symptoms of hypogonadism by ADAM questionnaire. This is a 10 question validated questionnaire focusing on key clinical feature of hypogonadism (Appendix).

\section{Material \& Methods}

All cases who had positive response based on decrease in loss of libido, strength of erections or any three nonspecific questions that included fatigability, mood changes and loss of height were subjected to biochemical investigations like serum Testosterone (total), LH , Prolactin, Thyroid function test, Hemogram, fasting Lipid profile, $\mathrm{HbAlc}$, Serum Creatinine and serum prostate specific antigen(PSA).

Patients with serum LH level in the low or normal range $(\leq 9.4$ $\mathrm{IU} / \mathrm{ml}$ ) and Testosterone $<300 \mathrm{ng} / \mathrm{dl}$ (Lower than Normal reference range 300-1000 ng/dl of our laboratory) were informed of their candidacy for treatment. The subjects were divided into two groups, Group I with serum Testosterone $<200 \mathrm{ng} / \mathrm{dl}$ were given Testosterone intramuscular injection monthly for 3 months and Group II with S. testosterone between 200- $300 \mathrm{ng} / \mathrm{dl}$ were given Clomiphene citrate (CC) $25 \mathrm{mg}$ daily PO for 3 months. All patients received counseling on diet and exercise consistent with American Diabetes Association recommendations throughout the study. Each of the participants was given verbal information and were asked to report if they experienced any side effect related to the use of treatment. The post treatment hormone estimation along with ADAM questionnaire value was conducted 3 months after commencing treatment. The study was approved by the local Ethics committee.

Special Investigations - Total testosterone (twice) and LH was measured by chemiluminescence immunoassay (Beckman Coulter, USA). In patient with diabetes, blood sample was collected always between $8 \mathrm{a} . \mathrm{m}$. and $10 \mathrm{am}$ in the fasting state. Serum was obtained by centrifugation and stored at $-20^{\circ} \mathrm{C}$ for assay in a batch.

Statistics -Data were collected, revised, coded, and entered in the statistical package for social science (IBM SPSS) version 16 . The qualitative data were presented as numbers and percentages while the quantitative data were presented as means, standard deviations. The comparison between two groups with qualitative data was done by using Chi-square test while the comparison between two groups with quantitative data and parametric distribution were done by using independent $\mathrm{t}$-test. Spearman correlation coefficients were used to assess the significance between two quantitative parameters in the same group. The confidence interval was set to $95 \%$ and the margin of error accepted was set to $5 \%$. P-value was considered significant at the level of $<0.05$.

\section{Results}

The baselisne characteristics of the study population are shown in Table 1. Both the groups were comparable. All subjects were between 40 to 70 years of age and mean age was $49.52 \pm 6.64$ years (SD). Duration of diabetes was more in group 2 in comparison to group 1 $(7.76 \pm 2.97$ vs $6.36 \pm 3.28, p=0.12)$. Both the groups were comparable at baseline for PSA, LH and HbA1c ( $\mathrm{p}$ value $>0.05$ ) while the mean testosterone level for group 1 was $279.68 \pm 20.23$ (range 257.45-299.91 $\mathrm{ng} / \mathrm{dl}$ ) and for group 2 was $158.69 \pm 39.04$ (range 62.87-199.7), $\mathrm{p}$ value $<0.001$. Mean ADAM score was significantly higher in group 2 in comparison to group $1(8.36 \pm 0.64$ vs $7.52 \pm 1.36)$. \{Table 2$\}$.

Table 1: Baseline Characteristics of Study Population.

\begin{tabular}{|l|c|c|c|}
\hline Characteristics & Group 1 (CC) N=40 & Group 2 (TT) N=32 & p-value \\
\hline Age & $47.92 \pm 6.64$ & $51.12 \pm 6.37$ & 0.088 \\
\hline Height $(\mathrm{cm})$ & $165.38 \pm 5.65$ & $165.34 \pm 5.36$ & 0.980 \\
\hline Weight $(\mathrm{kg})$ & $71.52 \pm 10.90$ & $71.68 \pm 7.98$ & 0.952 \\
\hline BMI $(\mathrm{kg} / \mathrm{m} 2)$ & $26.16 \pm 3.14$ & $26.25 \pm 2.35$ & 0.905 \\
\hline Waist circumference $(\mathrm{cm})$ & $94.06 \pm 8.09$ & $92.96 \pm 6.07$ & 0.589 \\
\hline Hip circumference $(\mathrm{cm})$ & $95.41 \pm 4.92$ & $93.77 \pm 3.81$ & 0.192 \\
\hline Waist Hip Ratio & $0.97 \pm 0.05$ & $0.99 \pm 0.05$ & 0.632 \\
\hline Diabetes duration (years) & $6.36 \pm 2.97$ & $7.76 \pm 3.28$ & 0.120 \\
\hline Hemoglobin (gm\%) & $13.72 \pm 0.82$ & $13.08 \pm 0.80$ & 0.009 \\
\hline Prolactin $(\mathrm{ng} / \mathrm{ml})$ & $7.22 \pm 3.22$ & $7.16 \pm 2.40$ & 0.939 \\
\hline TSH (mU/l) & $2.66 \pm 1.32$ & $2.68 \pm 1.05$ & 0.979 \\
\hline Hematocrit $(\%)$ & $43.22 \pm 1.85$ & $42.26 \pm 1.39$ & 0.042 \\
\hline S.Creatinine (mg/dl) & $0.98 \pm 0.19$ & $1.00 \pm 0.13$ & 0.750 \\
\hline PSA & $0.95 \pm 0.58$ & $0.79 \pm 0.30$ & 0.233 \\
\hline HbAlc & $8.91 \pm 1.29$ & $9.31 \pm 1.68$ & 0.354 \\
\hline ADAM & $7.52 \pm 1.36$ & $8.36 \pm 0.64$ & 0.007 \\
\hline Testosterone & $279.68 \pm 20.23$ & $158.69 \pm 39.04$ & 0.000 \\
\hline LH & $4.49 \pm 1.17$ & $4.53 \pm 0.88$ & 0.912 \\
\hline
\end{tabular}

After treatment for 3 months with Clomiphene Citrate and testosterone in group 1 and 2 respectively there was significant reduction in ADAM score in comparison to baseline. (7.52 \pm 1.36 at baseline to $2.68 \pm 0.90$ after 3 months in group $1, \mathrm{p}<0.001 ; 8.36 \pm 0.64$ at baseline to $3.24 \pm 0.83$ after 3 months in group $2, \mathrm{p}<0.001$ ). Similarly there was a significant improvement in mean testosterone levels in both the groups after 3 months of treatment $(279.68 \pm 20.23 \mathrm{ng} / \mathrm{dl}$ to $550.16 \pm 85.05$ in group $1, \mathrm{p}<0.001 ; 158.68 \pm 39.04$ to $509.72 \pm 39.18$ in group $2, \mathrm{p}<0.001)$. $\{$ Table 2$\}$

\section{Discussion}

The type $2 \mathrm{DM}$ has been recognized as a risk factor for male hypogonadism by most of the international endocrinology and andrology societies in their recommendations. ${ }^{[8]}$ The hypogonadism in diabetic male has been defined on the basis of serum total testosterone in most of the studies but clinical symptoms of hypogonadism have been rarely considered in combination with testosterone deficiency. ${ }^{[9]}$ In the present study, we used combination of both clinical (ADAM) and biochemical androgen deficiency to define hypogonadism.

Epidemiological ${ }^{[8]}$ studies have reported testosterone deficiency in $30 \%-50 \%$ of men with T2DM and up to $75 \%$ of them having sexual dysfunction. In our study, it was found that $118(47.2 \%)$ men had symptoms of androgen deficiency, while $72(28.8 \%)$ men had both symptoms and biochemical testosterone deficiency.

Our study showed that treatment of hypogonadism with Clomiphene citrate in comparison to testosterone therapy had similar degree of improvement in both clinical (ADAM) and biochemical 
Table 2: Analysis of group 1 (Clomiphene Citrate) group II (testosterone treated) patients before and after treatment:

\begin{tabular}{|l|c|c|c|c|c|c|}
\hline & \multicolumn{3}{|c|}{ Group I (CC) N=40 } & \multicolumn{3}{c|}{ Group II (TT) N=32 } \\
\hline \multicolumn{1}{|c|}{ Parameters } & Pre Mean \pm SD & Post Mean \pm SD & p-value & Pre Mean \pm SD & Post Mean \pm SD & p-value \\
\hline Hematocrit & $43.22 \pm 1.85$ & $43.99 \pm 1.95$ & 0.016 & $42.26 \pm 1.39$ & $43.26 \pm 0.93$ & $<0.001$ \\
\hline PSA & $0.95 \pm 0.58$ & $0.99 \pm 0.52$ & 0.153 & $0.79 \pm 0.29$ & $0.856 \pm 0.28$ & 0.044 \\
\hline Creatinine & $0.98 \pm 0.19$ & $0.99 \pm 0.16$ & 0.799 & $1.00 \pm 0.13$ & $0.99 \pm 0.13$ & 0.829 \\
\hline HbAlc & $8.92 \pm 1.29$ & $8.14 \pm 0.98$ & $<0.001$ & $9.31 \pm 1.67$ & $8.83 \pm 1.23$ & 0.001 \\
\hline LH & $4.49 \pm 1.17$ & $7.49 \pm 1.60$ & $<0.001$ & $4.52 \pm 0.87$ & $4.03 \pm 0.74$ & $<0.001$ \\
\hline ADAM score & $7.52 \pm 1.36$ & $2.68 \pm 0.90$ & $<0.001$ & $8.36 \pm 0.64$ & $3.24 \pm 0.83$ & $<0.001$ \\
\hline Total testosterone & $279.68 \pm 20.23$ & $550.16 \pm 85.05$ & $<0.001$ & $158.68 \pm 39.04$ & $509.72 \pm 39.18$ & $<0.001$ \\
\hline
\end{tabular}

(total testosterone) parameters. Clomiphene Citrate has been evaluated in hypogonadal patients ${ }^{[10]}$ but this is the first study to compare Clomiphene with testosterone in male T2DM patients with hypogonadism.

Therapy in both the groups was shown to improve glycemic control. This observation is in line with previous studies. ${ }^{[11]}$ Not surprisingly, treatment of hypogonadism has a significant positive impact on the health related quality of life in affected men. None of our study subjects in both the groups had reported any major side effect which required change/discontinuation of treatment.

The strength of our study was a prospective design assessing both the clinical response based on ADAM questionnaire as well as the biochemical response based on serum total testosterone levels. The weaknesses of our study included reasonable but small number of patients, short term follow up, lack of estimation of free testosterone and SHBG levels and limitations associated with ADAM questionnaire.

\section{Conclusions}

Treatment of secondary hypogonadism with Clomiphene Citrate in male patients with T2DM showed improvement in both clinical and biochemical measures of a similar degree and a tolerability profile that did not differ from that of testosterone therapy. It might be considered as an effective and safe alternative treatment strategy in secondary hypogonadal diabetic patients. Further long-term studies in a large cohort of patients with T2DM and hypogonadism are needed to evaluate the impact on metabolic parameters.

\section{References}

1. Gupta R, Misra A. (2007) Review: Type 2 diabetes in India: regional disparities. The British Journal of Diabetes \&amp; Vascular Disease 7(1):12-6.

2. Reaven GM. Banting lecture 1988. Role of insulin resistance in human disease. Diabetes 37(12):1595-607.[cross-ref]

3. Mulligan T, Frick MF, Zuraw QC, Stemhagen A, Mcwhirter C. et al.(2008) Prevalence of hypogonadism in males aged at least 45 years: the HIM study. International Journal of Clinical Practice 60(7):762-9. [cross-ref]

4. Dhindsa S, Prabhakar S, Sethi M, Bandyopadhyay A, Chaudhuri A, Dandona P et al.(2004) Frequent Occurrence of Hypogonadotropic Hypogonadism in Type 2 Diabetes. The Journal of Clinical Endocrinology \& Metabolism 89(11):5462-5468. [crossref]
5. Morley JE, Perry III HM, Kevorkian RT, Patrick P. et al.(2006) Comparison of screening questionnaires for the diagnosis of hypogonadism. Maturitas 53:424-9. [cross-ref]

6. Tajar A, Forti G, O'Neill T, Lee D, Silman A, Finn J et al.(2010) Characteristics of Secondary, Primary, and Compensated Hypogonadism in Aging Men: Evidence from the European Male Ageing Study. The Journal of Clinical Endocrinology \& Metabolism 95(4):1810-1818. [cross-ref]

7. Moskovic D, Katz D, Akhavan A, Park K, Mulhall J. et al.(2012) Clomiphene citrate is safe and effective for long-term management of hypogonadism. BJU International 110(10):1524-1528. [cross-ref]

8. Corona G, Monami M, Rastrelli G, Aversa A, Sforza A, Lenzi A et al.(2010) Type 2 diabetes mellitus and testosterone: a meta-analysis study. International Journal of Andrology 34(6pt1):528-540. [cross-ref]

9. Kapoor D, Aldred H, Clark S, Channer KS, Jones TH. et al.(2007) Clinical and biochemical assessment of hypogonadism in men with type 2 diabetes: correlations with bioavailable testosterone and visceral adiposity. Diabetes Care 30:911-917.[crossref]

10. Guay A, Jacobson J, Perez J, Hodge M, Velasquez E. et al.(2003) Clomiphene increases free testosterone levels in men with both secondary hypogonadism and erectile dysfunction: who does and does not benefit?. International Journal of Impotence Research 15(3):156-165.[cross-ref]

11. Katz D, Nabulsi O, Tal R, Mulhall J. et al.(2011) Outcomes of clomiphene citrate treatment in young hypogonadal men. BJU International 110(4):573-578.

\section{APPENDIX 1: ADAM QUESTIONNAIRE:}

1. Do you have a decrease in libido (sex drive)?

2. Do you have a lack of energy?

3. Do you have a decrease in strength and/or endurance?

4. Have you lost height?

5. Have you noticed a decreased "enjoyment of life"?

6. Are you sad and/or grumpy?

7. Are your erections less strong?

8. Have you noted a recent deterioration in your ability to play sports?

9. Are you falling asleep after dinner?

10. Has there been a recent deterioration in your work performance?

This questionnaire is suggestive of the presence of $H G$ when the patient answers 'yes' to items 1 or 7 or when 3 or more questions are answered affirmatively.

ASK Singh, Jitendra Chouhan and Rujul Jain (2021) Clomiphene Citrate is a Safe and Effective Alternative to Testosterone Replacement in Male Hypogonadism with Type 2 Diabetes Mellitus. Endocrinol Diabetes Metab J Volume 5(1): 1-3. 